\title{
INFORMATION SYSTEM FRAMEWORK FOR INTEGRATED RISK ASSESSMENT FROM NATURAL DISASTERS
}

\author{
D. Velev ${ }^{1, *}$, P. Zlateva $^{2}$ \\ ${ }^{1}$ University of National and World Economy, Sofia, Bulgaria - dgvelev@unwe.bg \\ ${ }^{2}$ Institute of Robotics, Bulgarian Academy of Sciences, Sofia, Bulgaria - plamzlateva@abv.bg
}

KEY WORDS: Information system, Risk assessment, Risk identification, Risk analysis, Risk evaluation, GIS, Natural disasters

\begin{abstract}
:
In recent years around the world, and in particular in Bulgaria, there is a reported increase in the number and intensity of disasters caused by natural phenomena. An increase is observed in the adverse effects of disasters on social relations, economic growth and sustainable development of the country. The purpose of the paper is to propose a general framework of information system for integrated risk assessment from natural disasters with the help of modern information and communication technologies. Innovative and classical techniques (quantitative and qualitative approaches, deterministic and stochastic methods, intelligent methods as fuzzy logic and neural networks, etc.) are applied in the overall process of the risk assessment (IEC/ISO 31010:2009): risk identification (identification of single and multiple natural disasters), risk analysis (determination of the disaster probability/intensity; interdependence of multiple disasters; exposure; object vulnerabilities; social, ecological and economical consequences) and risk evaluation. The information system is designed on the principle of modules which implement interaction with geographical information system (GIS) and heterogeneous databases containing information about the monitored objects and potential natural disasters in Bulgaria. The information system will use cloud computing and online network for exchange of heterogeneous databases and expertise for the risks from natural disasters. The proposed information system could successfully assist all stakeholders about risk assessment of the monitored objects before, during or after the occurrence of natural disasters. This system could be considered as a unified platform for interdisciplinary research concerning natural disasters.
\end{abstract}

\section{INTRODUCTION}

In recent years around the world and in particular in Bulgaria there is a reported increase in the number and intensity of disasters caused by natural phenomena or human activities (Padli et. al., 2010; Pollner et. al., 2010; Prevention Web, 2018) An increase is observed in the adverse effects of disasters on social relations, economic growth and sustainable development of the country (Silva and Costa, 2018; UNISDR, 2017).

For this reason, the UN General Assembly, the European Commission, as well as the Bulgarian Parliament constantly develops and adopts legal documents to assist the responsible institutions and relevant organizations in their activities related to the effective reduction of the risk of disasters (OSCE, 2016; Oxfam International Secretariat, 2017; United Nations, 2016; UNISDR, 2009; UNISDR, 2016). In this respect, it is important to point out that on 18 March 2015 at the Third UN World Conference on Disaster Risk Reduction in Sendai City, Miyagi Prefecture, Japan, the UN Member States adopted the so-called Sendai Framework for Disaster Risk Reduction 2015-2030 (UNISDR, 2017). The Sendai Framework is a 15-year, voluntary, non-binding agreement which recognizes that the State has the primary role to reduce disaster risk but that responsibility should be shared with other stakeholders including local government, the private sector and other stakeholders. It aims for the following outcome: "The substantial reduction of disaster risk and losses in lives, livelihoods and health and in the economic, physical, social, cultural and environmental assets of persons, businesses, communities and countries" (United Nations Office for Disaster
Risk Reduction (UNISDR), Sendai Framework for Disaster Risk Reduction, https://www.unisdr.org/we/coordinate/sendaiframework).

In the working document of the European Commission SEC1626/2010 "Guidelines for risk assessment and mapping the regarding disaster risk management" offers rules, which focus on the processes of assessment and mapping of national risks, performing in the wider framework of risk management (European Commission, 2010). Our country Bulgaria has adopted a National Strategy for Disaster Risk Reduction 20142020. Now it is discussed draft National Strategy for Disaster Risk Reduction 2017-2030.

The geographical location, natural features and the economic situation predefine the high vulnerability of our country to diverse natural disasters, which may cause considerable human, environmental and material losses.

Certainly, in today's global climate change, the role of information and communication technologies is becoming an indispensable element in the systems of protecting the life, health and property of the population, environment and critical infrastructure. Internet technologies for disaster management are used in our country, but still insufficient. Actively on these issues, work in leading research centers in Europe, USA, Australia, etc. Various information systems are available that include a variety of risk assessment tools for natural disasters using databases and geographical information system (GIS).

It should be noted that the results of scientific and applied research on risk assessment mostly relate to one particular 
natural disaster. Insufficient number of authors investigates problems of multiple natural hazards and risks (Eshrati et. al., 2015; Harab et. al., 2017; ). However in most of the research, it is not sufficiently taken into account the joint impact of several disasters on society, environment and infrastructure (Kreibich et. al., 2014; Liu et. al., 2016). Usually, individual research teams use different tools (methods, models, databases, programming languages, software environments, etc.). For this reason, it is difficult to compare and mutually use the results and to determine the overall negative consequences from the investigated disasters.

Therefore, integrated risk assessment of natural disasters should be seen as a complex multidisciplinary process that requires the pooling of the efforts of specialists from different scientific areas. This fact predetermines the construction of an information system that includes different research tools and heterogeneous databases for the investigation of various disasters. This system can be successfully used as a unified platform for interdisciplinary research and knowledge sharing.

The paper purpose is to propose a general framework of information system for integrated risk assessment of natural disasters based on the standard IEC/ISO 31010:2010 and with the help of modern information technologies. The information system is designed on the principle of modules that implement interaction with GIS and heterogeneous databases.

\section{PROBLEM STATEMENT OF THE RISK ASSESSMENT}

The risk assessment is the core of the overall risk management process according to basic standards: ISO 31000:2009 "Risk management — Principles and guidelines" and IEC/ISO 31010:2009 "Risk management - Risk assessment techniques". This risk management process is presented in Fig. 1. It can be seen that the risk assessment includes risk identification, risk analysis and risk evaluation.

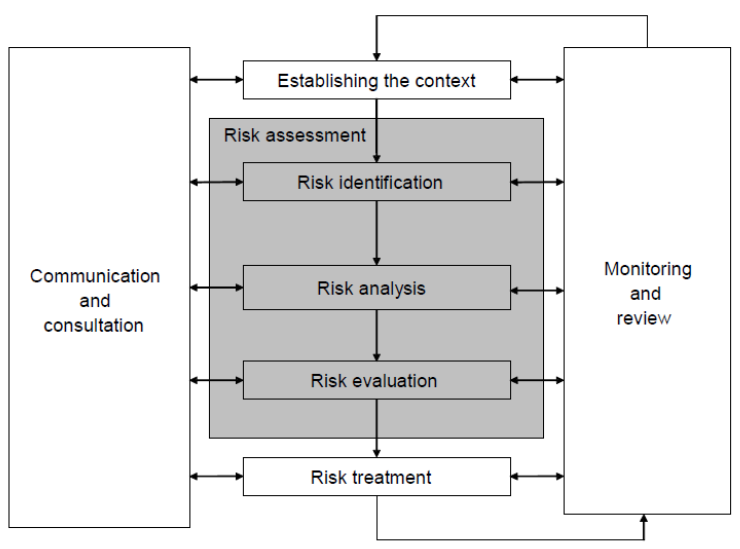

Figure 1. The risk assessment as part of the risk management

In this study for the purpose of risk assessment from natural disasters the following term definitions are used (UNISDR, 2009, IEC/ISO 31010, 2010):

- Hazard is a dangerous phenomenon, substance, human activity or condition that may cause loss of life, injury or other health impacts, property damage, loss of livelihoods and services, social and economic disruption, or environmental damage.
- Natural hazard: Natural process or phenomenon that may cause loss of life, injury or other health impacts, property damage, loss of livelihoods and services, social and economic disruption, or environmental damage. Natural hazard events can be characterized by their magnitude or intensity, speed of onset, duration, and area of extent.

- Hazard assessments determine the probability of occurrence of a certain hazard of certain intensity.

- Exposure: People, property, systems, or other elements present in hazard zones that are thereby subject to potential losses.

- Vulnerability: The characteristics and circumstances of a community, system or asset that make it susceptible to the damaging effects of a hazard. In probabilistic/quantitative risk assessments, the term vulnerability expresses the part or percentage of Exposure that is likely to be lost due to a certain hazard.

- Risk is a combination of the consequences of an event (Hazard) and the associated likelihood/probability of its occurrence.

- Risk assessment is the overall process of risk identification, risk analysis, and risk evaluation.

- Risk identification is the process of finding, recognizing and describing risks.

- Risk analysis is the process to comprehend the nature of risk and to determine the level of risk.

- Risk evaluation is the process of comparing the results of risk analysis with risk criteria to determine whether the risk and/or its magnitude is acceptable or tolerable.

- Risk criteria are the terms of reference against which the significance of a risk is evaluated.

- Consequences are the negative effects of a disaster expressed in terms of human impacts, economic and environmental impacts, and political/social impacts.

- Human impacts are defined as the quantitative measurement of the following factors: number of deaths, number of severely injured or ill people, and number of permanently displaced people.

- Economic and environmental impacts are the sum of the costs of cure or healthcare, cost of immediate or longer-term emergency measures, costs of restoration of buildings, public transport systems and infrastructure, property, cultural heritage, etc., costs of environmental restoration and other environmental costs (or environmental damage), costs of disruption of economic activity, value of insurance pay-outs, indirect costs on the economy, indirect social costs, and other direct and indirect costs, as relevant.

- Political/social impacts are usually rated on a semiquantitative scale and may include categories such as public outrage and anxiety, encroachment of the territory, infringement of the international position, violation of the democratic system, and social psychological impact, impact on public order and safety, political implications, psychological implications, and damage to cultural assets, and other factors considered important which cannot be measured in single units, such as certain environmental damage. 
- Threat is a potentially damaging physical event, phenomenon or activity of an intentional/ malicious character.

- Single-risk assessments determine the singular risk (i.e. likelihood and consequences) of one particular hazard (e.g. flood) or one particular type of hazard (e.g. flooding) occurring in a particular geographic area during a given period of time.

- Multi-risk assessments determine the total risk from several hazards either occurring at the same time or shortly following each other, because they are dependent from one another or because they are caused by the same triggering event or hazard; or merely threatening the same elements at risk (vulnerable/ exposed elements) without chronological coincidence.

According IEC/ISO 31010:2009 in situations where the likelihood of occurrence of a hazard of certain intensity can be quantified investigators refer to the term probability of occurrence. When the extent of the impacts is independent of the probability of occurrence of the hazard, which is often the case for purely natural hazards, such as earthquakes or storms, risk can be expressed algebraically as:

\section{Risk $=$ probability of occurrence $*$ hazard impact}

or

$$
\left.R=\mathrm{F}\left(p^{*} C\right) \quad \text { or } \quad R=p \cdot C\right),
$$

where $R$ is risk; $P$ - probability of occurrence of the natural hazard; $C$-consequences (natural hazard impact).

If there is enough information (quantitative and/or qualitativeexpert) for the vulnerability $-V$ and the exposure - $E$ of the critical infrastructures and their locations, then the risk $-R$ can be expressed with the following functional dependency:

$$
\text { Risk }=f\left(p^{*} E * V\right) \quad \text { or } \quad R=p . E . V
$$

Risk matrix is very helpful in the risk assessment process. In particular the risk matrix or so-called consequence/ probability matrix is a means of combining qualitative or semi-quantitative ratings of consequence and probability to produce a level of risk. The format of the risk matrix depends on the context in which it is used. The scale used may have 5 or more points. The matrix may be set up to give extra weight to the impact or to the likelihood, or it may be symmetrical.

Usually in risk assessment process the risk matrix $5 \times 5$ is used (Fig. 2). Here the Probability levels (Relative likelihood) are graded as "Very low", "Low", "Medium", "High" and "Very high".

Consequences (Relative impact) are also graded as "Very low", "Low", "Medium", "High" and "Very high". The Risk levels, $R$ are defined as "Low", "Medium", "High" and "Very high".

The following three main types of impacts in regard to the risk assessment are defined: human, economic/environmental, political/social. Within each category of impact (human, economic/environmental, political/social) the relative importance can be graded using a single set of criteria to score the relative likelihood and the relative impact applicable to the different hazards or risk scenarios.
In particular, the human impact can be estimated in terms of number of affected people and the economic/ environmental impact can be measured in currency (for example in Euro). The political/ social impact can be measured in a qualitative/ semi-quantitative scale comprising a number of classes, for example five classes as (1) limited/ insignificant, (2) minor/ substantial, (3) moderate/ serious, (4) significant/ very serious, (5) catastrophic/ disastrous.

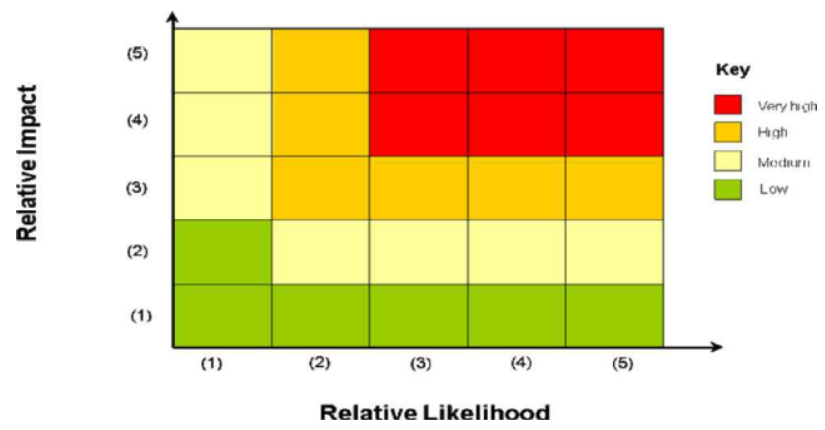

Figure 2. Risk matrix 5x5: Consequences (Relative impact) / Probability (Relative likelihood).

The overall risk assessment requires producing distinct risk matrices for human impact, economic / environmental impact and political/social impact. However it is necessary to point that these categories are measured with distinct scales. For this reason it is very difficult to compare.

\section{ESSENCE OF THE INTEGRATED RISK ASSESSMENT FROM NATURAL DISASTERS}

The integrated risk assessment includes the three stages (risk identification, risk analysis, risk evaluation) as functional elements (modules) in the proposed information system.

\subsection{Risk identification of the monitored object from natural disasters - module 1}

According to the above definition, risk identification is the process of finding, recognizing and describing risks. In cases where the risk is induced by natural disasters, then the process of risk identification requires descriptions of all potential natural hazards, which cause these disasters.

In this paper, the risk identification of Bulgarian electricity transmission and distribution network requires descriptions of the natural hazards occurring on the territory of the country. The predominant natural hazards on Bulgarian territory are identified as follows:

- Geological processes and phenomena: Earthquakes; Slope failures (landslides, landslips, creep, falls, flows, subsidence); Mud-rock flows (seli); Erosion and abrasion; Storm surge.

- Hydrological processes and phenomena: Floods; Dry periods; Snow flows and glaciations; Icings.

- Meteorological processes and phenomena: Strong wind; Extreme temperatures; Freezings, Drought, Tornado phenomena; Dust storms; Hailstorms; Wet snow; Fog (coastal, evaporation, radiation, valley, upslope); Thunderstorm; Silver thaw; Wild land fire. 


\subsection{Risk analysis of the monitored object from natural} disasters - module 2

Risk analysis is the process to cover the nature of the risk and determine the risk level. For each risk and risk scenario set in the previous stage of risk identification, the risk analysis process carries out a detailed (and if possible a quantitative) evaluation of the probability of its occurrence and the severity of the potential impacts.

The risk analysis, when possible, is based on quantitative information:

- The assessment of the likelihood of a danger must be based upon the ability of the historical frequency of event at a similar scale and the available statistical information in terms of the analysis of the underlying mechanisms of action.

- The assessment of the level of impact should be quantitative and objective.

The following main risk activities should be taken into account in the overall process of the risk analysis:

- Hazard analysis

- Geographical Analysis (location, range);

- Temporal analysis (frequency, duration, etc..);

- Analysis of the size (scale, intensity);

- Probability of occurrence.

- Vulnerability analysis

- Identification of elements (monitored object) at risk;

- Identification of the factors for the vulnerabilities/impacts (on people, economy, environment, society);

- Assessment of the probable impact;

- Analysis of ability to reduce exposure and/or vulnerability.

The economic losses are assessed, which allows you to analyze both the tangible and intangible damage from disasters. In this sense, the economic analysis to assess the potential losses leads to widening the scope of the assessment. According to the accepted classification there are tangible and intangible losses that could additionally be grouped into of direct and indirect.

In this study, the main task of the module 2 for the risk analysis is consists in the determination of the levels of risk for the monitored object from natural disasters.

In this study, it is considered that the natural hazard that causes the corresponding natural disaster has four levels of intensity. Furthermore it is necessary to note that the adequate risk analysis requires producing distinct risk matrices for each intensity levels of the natural hazard. Usually it is considered the following four levels of the natural hazard intensity: (1) Low hazard intensity, (2) Medium hazard intensity, (3) High hazard intensity, (4) Very high hazard intensity (Table 1).

\begin{tabular}{|c|c|}
\hline Relative intensity & Hazard intensity levels, $H$ \\
\hline 4 & Very high hazard intensity \\
3 & High hazard intensity \\
2 & Medium hazard intensity \\
1 & Low hazard intensity \\
\hline
\end{tabular}

Table 1. Hazard intensity levels
In this case, the risk is defined as follow:

$$
R=\sum_{k=1}^{4} R_{k}
$$

where $R_{k}$ is the determined risk corresponding to $k$ intensity level of natural hazard, $k=1, \ldots, 4$.

The risk $R_{k}$ is obtained by following product

$$
R_{k}=P_{k} \cdot C_{k} \quad k=1, \ldots, 4
$$

where $P_{k}$ is the occurrence probability of the natural hazard with $k$ intensity level; $C_{k}$ is the consequences caused by action of the of the natural hazard with $k$ intensity level.

The calculated value of consequences $C_{k}$ and the given value of the probability $P_{k}$ for occurrence of the natural hazard with $k$ intensity level in the considered time interval are substituted in (2) to calculated corresponding risk level $R_{k}$. Than using (1) it is calculated the total risk assessment $R$ of the monitored object from the natural disasters in a given geographic region from natural hazard with four intensity levels for a certain time interval.

Each of the resulting risk levels $R$ and $R_{k}, k=1, \ldots, 4$ can be presented as a separate risk matrix as the proposed on Fig. 2.

The risk matrix parameters (Risk level, Relative likelihood and Relative impact) can be determined as follows:

- First from Table 2 it can be determined the particular level of the probability $P_{k}$ by using predefined range of each of the five levels. The constants $I P_{i}, \mathrm{i}=1, \ldots .5$ are previously defined on the base of the real data or expert knowledge.

- Second by analogy with the probability from Table 3 it can be determined the particular level of the consequences $C_{k}$ by using predefined range of each of the five levels. The constants $I C_{i}, \mathrm{i}=1, \ldots 5$ are previously given.

- Third from Table 4 for each of the resulting risk assessments $R$ and $R_{k}, k=1, \ldots, 4$, can be determined the particular risk levels by using predefined range of each of the four levels. The constants $I R_{i}, \mathrm{i}=1, \ldots 4$ are previously given.

The proposed risk assessment with these determinations of the particular risk levels $R$ and $R_{k}, \mathrm{k}=1, \ldots, 4$, leads to more effectiveness of the risk management about natural hazards.

\begin{tabular}{|c|c|c|}
\hline $\begin{array}{c}\text { Relative } \\
\text { likelihood }\end{array}$ & $\begin{array}{c}\text { Probability levels, } \\
P_{k}\end{array}$ & $\begin{array}{c}\text { Probability value } \\
\text { intervals }\end{array}$ \\
\hline 1 & Very low probability & $I P_{4}<P_{k}$ \\
2 & Low probability & $I P_{3}<P_{k} \leq I P_{4}$ \\
3 & Medium probability & $I P_{2}<P_{k} \leq I P_{3}$ \\
4 & High probability & $I P_{1}<P_{k} \leq I P_{2}$ \\
5 & Very high probability & $P_{k} \leq I P_{1}$ \\
\hline
\end{tabular}

Table 2. Relative likelihood / Probability levels

\subsection{Risk evaluation of the monitored object from natural disasters - module 3}

The risk evaluation is carried out by comparing the determined risk levels according Table 4 with specific risk criteria to decide whether the risk is acceptable or inadmissible. 


\begin{tabular}{|c|c|c|}
\hline $\begin{array}{c}\text { Relative } \\
\text { impact }\end{array}$ & $\begin{array}{c}\text { Aggregated loss / } \\
\text { Consequences levels, } C_{k}\end{array}$ & $\begin{array}{c}\text { Consequence } \\
\text { value intervals }\end{array}$ \\
\hline 1 & Very low probability & $I C_{4}<C_{k}$ \\
2 & Low probability & $I C_{3}<C_{k} \leq I C_{4}$ \\
3 & Medium probability & $I C_{2}<C_{k} \leq I C_{3}$ \\
4 & High probability & $I C_{1}<C_{k} \leq I C_{2}$ \\
5 & Very high probability & $C_{k} \leq I C_{1}$ \\
\hline
\end{tabular}

Table 3. The Consequences levels

\begin{tabular}{|c|c|}
\hline Risk levels, $R$ or $R_{k}$ & Risk value intervals \\
\hline Very high risk & $I R_{3}<R_{k}$ \\
High risk & $I R_{2}<R_{k} \leq I R_{3}$ \\
Medium risk & $I R_{1}<R_{k} \leq I R_{2}$ \\
Low risk & $R_{k} \leq I R_{I}$ \\
\hline
\end{tabular}

Table 4. The Risk levels

\section{AN APPROACH TO INTEGRATED RISK ASSESSMENT OF NATURAL HAZARDS THROUGH FUZZY LOGIC DISASTERS}

The idea is the proposed approach to integrated risk assessment to take into account quantitative and qualitative characteristics of all natural hazards in monitored object. The approach uses the fuzzy logic (Zimmerman, 1996).

This approach is on the based on module 4, which includes the following steps (Zlateva and Velev, 2013):

Step 1: The basic sets and subsets for risk level of the monitored object and severities of natural hazard are introduced and they are described in natural language:

a. Complete set of risk level of monitored object $R$ is divided into five subsets of the form:

$R_{1}$ - subset "Very low level of risk";

$R_{2}$ - subset "Low level of risk";

$R_{3}$ - subset "Middle level of risk";

$R_{4}$ - subset "High level of risk";

$R_{5}$ - subset "Very high level of risk".

b. Complete set of severity of natural hazard $H$ is divided into five subsets of the form:

$V S$ - subset "Very small severity of natural hazard";

$S$ - subset "Small severity of natural hazard";

$M$ - subset "Middle severity of natural hazard";

$B$ - subset "Big severity of natural hazard";

$V B$ - subset "Very big severity of natural hazard".

Here and below it is assumed that the all elements of set $R$ and $D$ accept values in the interval $[0,10]$.

Step 2: The natural hazards (risk indicators) $H=\left\{H_{i}\right\}$, $i=1, \ldots, n$, which are typical for monitored object, are determined.

Step 3: The corresponding degree of importance in the risk analysis $\lambda_{i}$ is assigned to each natural hazard $H_{i}$. In order to appreciate this degree, it is necessary to arrange all the hazards in decreasing importance so as to satisfy the rule

$$
\lambda_{1} \geq \lambda_{2} \geq \ldots \geq \lambda_{n}>0 \quad \text { and } \quad \sum_{i=1}^{n} \lambda_{i}=1
$$

If all indicators are equal importance, then

$$
\lambda_{i}=\frac{1}{n}, \quad i=1, \ldots, n .
$$

Step 4: A classification of the current value $r$ of the level of risk as a criterion to split the set $R$ into fuzzy subsets is constructed (Table 5).

Step 5: The membership function "severity of natural hazard" for each value of hazard variable $H$ is calculated

Each hazard variable $H_{i}, i=1, \ldots, n$ has a corresponding membership function $\mu_{i j}, j=1, \ldots, 5$ to the five fuzzy subsets.

The membership functions $\mu_{i j}$ are defined with the following formulae:

$$
\begin{aligned}
& \mu_{i 1}=\left\{\begin{array}{rr}
1, & 0 \leq H_{i}<1.5 \\
2.5-H_{i}, & 1.5 \leq H_{i}<2.5 \\
0, & 2.5 \leq H_{i} \leq 10
\end{array}\right. \\
& \mu_{i 2}=\left\{\begin{array}{rr}
0, & 0 \leq H_{i}<1.5 \\
H_{i}-1.5, & 1.5 \leq H_{i}<2.5 \\
1, & 2.5 \leq H_{i}<3.5 \\
4.5-H_{i}, & 3.5 \leq H_{i}<4.5 \\
0, & 4.5 \leq H_{i} \leq 10
\end{array}\right. \\
& \mu_{i 3}=\left\{\begin{array}{rr}
0, & 0 \leq H_{i}<3.5 \\
H_{i}-3.5, & 3.5 \leq H_{i}<4.5 \\
1, & 4.5 \leq H_{i}<5.5 \\
6.5-H_{i}, & 5.5 \leq H_{i}<6.5 \\
0, & 6.5 \leq H_{i} \leq 10
\end{array}\right. \\
& \mu_{i 4}=\left\{\begin{array}{rr}
H_{i}-7.5, & 7.5 \leq H_{i}<8.5 \\
1, & 8.5 \leq H_{i} \leq 10 \\
0, & 0 \leq H_{i}<5.5 \\
H_{i}-5.5, & 5.5 \leq H_{i}<6.5 \\
1, & 6.5 \leq H_{i}<7.5
\end{array}\right.
\end{aligned}
$$

It are carried out the calculation of the values of the five membership functions "severity of natural hazard" $\mu^{k} i j$ in regard to each of the natural hazard $H_{i}, \quad i=1, \ldots, n$ for each of the monitoring regions $X_{k}, k=1, \ldots, m$. 
The results are presented in tables for each of the natural hazard, as shown in Table 6.

\begin{tabular}{|c|c|c|}
\hline $\begin{array}{ll}\text { Risk } & \text { value } \\
\text { interval, } r\end{array}$ & $\begin{array}{l}\text { Classification } \\
\text { of the risk } \\
\text { level, } R_{i}\end{array}$ & $\begin{array}{l}\text { Membership } \\
\text { function of the } \\
\text { risk level, } \mu_{i}\end{array}$ \\
\hline $0 \leq r \leq 1.5$ & $R_{1}$ & 1 \\
\hline $1.5<r<2.5$ & $\begin{array}{l}R_{1} \\
R_{2}\end{array}$ & $\begin{array}{l}\mu_{l}=2.5-r \\
1-\mu_{l}=\mu_{2}\end{array}$ \\
\hline $2.5 \leq r \leq 3.5$ & $R_{2}$ & 1 \\
\hline $3.5<r<4.5$ & $\begin{array}{l}R_{2} \\
R_{3}\end{array}$ & $\begin{array}{l}\mu_{2}=4.5-r \\
1-\mu_{2}=\mu_{3}\end{array}$ \\
\hline $4.5 \leq r \leq 5.5$ & $R_{3}$ & 1 \\
\hline $5.5<r<6.5$ & $\begin{array}{l}R_{3} \\
R_{4}\end{array}$ & $\begin{array}{l}\mu_{3}=6.5-r \\
1-\mu_{3}=\mu_{4}\end{array}$ \\
\hline $6.5 \leq r \leq 7.5$ & $R_{4}$ & 1 \\
\hline $7.5<r<8.5$ & $\begin{array}{l}R_{4} \\
R_{5}\end{array}$ & $\begin{array}{l}\mu_{4}=8.5-r \\
1-\mu_{4}=\mu_{5}\end{array}$ \\
\hline $8.5 \leq r \leq 10$ & $R_{5}$ & 1 \\
\hline
\end{tabular}

Table 5. Risk level classification of monitoring region

Step 6: The value $r$ of the "level of risk" in regard to all the considered natural hazards for each of the monitoring regions is calculated

The value $r_{k}$ of the "level of risk" in regard to all the considered natural hazards $H_{i}, \quad i=1, \ldots, n$ for each of the monitoring regions $X_{k}, k=1, \ldots, m$ are determined as follows

$r_{k}=\sum_{j=1}^{5} \alpha_{j} \sum_{i=1}^{n} \lambda_{i} \mu^{k}{ }_{i j}=\sum_{j=1}^{5} \alpha_{j} q_{j}^{k} \quad q_{j}=\sum_{i=1}^{5} \lambda_{i} \mu_{i j}$

A node point vector

$$
\alpha=\left(\alpha_{1}, \alpha_{2}, \alpha_{3}, \alpha_{4}, \alpha_{5}\right)
$$

is introduced. In this investigation the node point vector has following elements

$$
\alpha=(1,3,5,7,9) \text {. }
$$

\begin{tabular}{|c|c|c|c|c|c|c|}
\hline \multirow[t]{2}{*}{ No } & \multirow{2}{*}{$\begin{array}{l}\text { Monitored } \\
\text { object, } X\end{array}$} & \multicolumn{5}{|c|}{ Membership functions of $H_{i}$} \\
\hline & & $V S$ & $S$ & $M$ & $B$ & $V B$ \\
\hline 1. & $X_{1}$ & $\mu_{i 1}^{1}$ & $\mu_{i 2}^{1}$ & $\mu_{i 3}^{1}$ & $\mu_{i 4}^{1}$ & $\mu_{i 5}^{1}$ \\
\hline 2. & $\cdots$ & & & & & \\
\hline 3. & $X_{k}$ & $\mu^{k}{ }_{i 1}$ & $\mu^{k}{ }_{i 2}$ & $\mu^{k}{ }_{i 3}$ & $\mu^{k}{ }_{i 4}$ & $\mu^{k}{ }_{i 5}$ \\
\hline $\begin{array}{l}4 . \\
5\end{array}$ & $\ldots$ & & & & & \\
\hline 5. & & $\mu^{m}{ }_{i 1}$ & $\mu^{m}{ }_{i 2}$ & $\mu^{m}{ }_{i 3}$ & $\mu^{m}{ }_{i 4}$ & $\mu^{m}{ }_{i 5}$ \\
\hline
\end{tabular}

Table 6. Membership functions of $H_{i}$ for monitored object

Step 7: The linguistic classification of the risk level of monitored objects about all the considered natural hazards is carried out.

The calculated value $r$ of the variable "level of risk" is classifies on the basis of the data in Table I.

The main result of the classification is linguistic description of the risk level of monitored object $R_{i}$ in regard to all the considered natural hazards. Additional result is the degree of expert certainty in the correctness of the classification, which is given by value of corresponding membership function $\mu_{i}$.
Thus the conclusion about "level of risk" acquires not only linguistic form, but also characterization for the reliability of this assertion.

\section{MAIN ELEMENTS OF THE GENERAL FRAMEWORK OF THE INFORMATION SYSTEM}

Modern research has shown the need to integrate the efforts of interdisciplinary teams from various countries for the development of information systems for emergency management, including modules for the risk analysis and assessment from natural disasters (Zlateva et. al, 2013).

The data used in the risk assessment from natural disasters usually include geographic data for the damage area, data about the possible buildings for temporary accommodation and transport data available for victims and aid workers available for rescue operations, and multiple measurements in the field. The data may belong to different autonomous organizations, such as government organizations, non-governmental organizations, international non-governmental organizations, individuals, communities and industries.

Therefore, in addition to integrating and managing data from different organisations, it is necessary to coordinate these organisations with which to implement effective communication and cooperation with the development of Web 2.0 technologies, such as Social Network Sites (SNS), blogs, wikis, and video sharing, a broad community of users they are able to interact or collaborate with one another in dialogue via social media as creators of content generated by users in a virtual environment (Gartner, 2012; Srikanth, 2012., Ubiquity of mobile wireless devices facilitate the participation of the general public in the production, distribution and consumption of information anywhere and anytime.

Cloud Computing can provide data and communications as a service to management of emergencies (Velev, 2015). Cloud system for disaster management can provide a special platform that allows users (employees, first responders, local non-profit organizations to work in conditions of disaster, volunteers and locals) for the organization of access to information, communication and collaboration in real time from all types of computing devices, including mobile, portable devices such as smartphones, tablets, iPpad, etc. (Velev, 2014; Zong et.al.2016). Such a system could help for the creation of efficient and scalable cloud environment in which a diverse set of organizations can share their data, knowledge, experience, and computing resources for coping with natural disasters.

\section{CONCLUSION}

The paper purpose is to propose a general framework of information system for integrated risk assessment of natural disasters based on the standard IEC/ISO 31010:2010 and with the help of modern information technologies. The information system is designed on the principle of modules that implement interaction with GIS and heterogeneous databases.

A framework of information system for integrated risk assessment of natural disasters based on the standard IEC/ISO 31010:2009 is proposed. Innovative and classical techniques are applied in the overall process of the risk assessment (IEC/ISO 31010:2009): risk identification (identification of single and multiple natural disasters), risk analysis (determination of the disaster probability/intensity; 
interdependence of multiple disasters; exposure; object vulnerabilities; social, ecological and economical consequences) and risk evaluation.

It is suggested the the information system to be designed on the principle of modules which implement interaction with geographical information system (GIS) and heterogeneous databases containing information about the monitored objects and potential natural disasters in Bulgaria. It is used the cloud computing and online network for exchange of heterogeneous databases and expertise for the risks from natural disasters.

The proposed information system could successfully assist all stakeholders about risk assessment of the monitored objects before, during or after the occurrence of natural disasters. This system could be considered as a unified platform for interdisciplinary research concerning natural disasters.

\section{ACKNOWLEDGEMENT}

The authors wish to thank the Bulgarian National Science Fund for the partial financial support under the Grant № DFNI-I02/15 from 12.12.2014, titled "Information System for Integrated Risk Assessment from Natural Disasters".

\section{REFERENCES}

Eshrati, L.; Mahmoudzadeh, M.; Taghvaei, M.; 2015. Multi hazards risk assessment, a new methodology. Int. Journal of Health System and Disaster Management, 3 (2), pp. 79-88.

European Commission 2010 Risk Assessment and Mapping Guidelines for Disaster Management, working paper.

Gartner, 2012. The Nexus of Forces: Social, Mobile, Cloud and Information,

http://www.gartner.com/DisplayDocument?doc_cd=234840.

Harab, M. M. and Dell'Acqua, F., 2017. Remote Sensing in Multi-Risk Assessment: Improving disaster preparedness. IEEE Geoscience and Remote Sensing Magazine, 5(1), pp. 53-65.

IEC/ISO 31010:2009 Risk management - Risk assessment techniques.

ISO 31000:2009 Risk management - Principles and guidelines.

Kreibich, H.; Bubeck, P.; Kunz, M.; Mahlke, H.; Parolai, S.; Khazai, B.; Daniell, J.; Lakes, T.; Schroter, K., 2014. A review of multiple natural hazards and risks in Germany. Nat Hazards, 74, pp. 2279-2304.

Liu, B.; Siu, Y. L.; Mitchell, G.; Xu, W., 2016. The danger of mapping risk from multiple natural hazards. Nat Hazard, 82, pp. 139-153, doi 10.1007/s11069-016-2184-5

Organization for Security and Co-operation in Europe (OSCE) 2016 Protecting Electricity Networks from Natural Hazards, Vienna, Austria.

Oxfam International Secretariat, 2017. Community based disaster risk management minimum standards, CI, https://www.preventionweb.net/publications/view/57073
Padli, J.; Habibullah, M. and Baharom, A. 2010. Economic impact of natural disasters' fatalities, International Journal of Social Economics, 37(6) pp. 429 - 441.

Pollner, J.; Kryspin-Watson, J. and Nieuwejaar, S. 2010. Disaster Risk Management and Climate Change Adaptation in Europe and Central Asia. Global Facility for Disaster Reduction and Recovery: The World Bank.

Prevention Web, 2018 - Strengthening climate change adaptation through effective disaster risk reduction, http://www.preventionweb.net

Silva, M. and Costa J., 2018. Urban floods and climate change adaptation: the potential of public space design when accommodating natural processes. Water, 10(2), pp. 180-191

Srikanth, A., 2012., Social media can solve many problems during natural disasters, http://infworm.com/

$\begin{array}{lcccc}\text { UNISDR } 2009 & \text { UN } & \text { International } & \text { Strategy for } & \text { Disaster } \\ \text { Reduction } & \text { Sec, } & 15 & \text { January } & 2009, \\ \text { www.unisdr.org/eng/library/lib-terminology-eng.htm. } & \end{array}$
www.unisdr.org/eng/library/lib-terminology-eng.htm.

United Nations, 2016, UN Plan of Action on Disaster Risk Reduction for Resilience: Towards a Risk informed and Integrated Approach to Sustainable Development, 2016. https://www.preventionweb.net/publications/view/49076

United Nations Office for Disaster Risk Reduction (UNISDR) 2015 Sendai Framework for Disasster Risk reduction 20152030, Proceedings Third UN Word Conference on Disaster Risk Reduction, 14-18 March 2015, Sendai, Japan.

United National Office for Disaster Risk Reduction (UNISDR) 2016. Strategic Framework 2016-2021, https://www.unisdr.org/who-we-are/programme-and-reports

United National Office for Disaster Risk Reduction (UNISDR) 2017. Annual report 2016, https://www.unisdr.org/

Velev, D., 2015. Security Aspects of Cloud-Based Mobile Learning, Financial and credit activity: problems of theory and practice, 2 (17), pp. 240-251.

Velev, D., 2014. Challenges and Opportunities of Cloud-Based Mobile Learning, Int. Journal of Information and Education Technology, 4 (1), pp. 49-53.

Zimmerman, H., 1996. Fuzzy set theory and it applications. Kluwer Academic Publishers, Norwell MA, USA.

Zlateva, P. and Velev, V., 2013. Complex risk analysis of natural hazards through fuzzy logic. Journal of Advanced Management Science, 1 (4), pp. 395-400.

Zlateva P.; Hirokawa, Y.; Velev, D., 2013. An Integrated Approach for Risk Assessment of Natural Disasters Using Cloud Computing, International Journal of Trade, Economics and Finance, 4 (3), pp. 134-138.

Zong X., Q. Li, Zh. Yang, K. He, D. Velev 2016. Comprehensive Management Platform of Natural Disasters Based on Cloud Computing, International Journal of Machine Learning and Computing, 6 (3), pp. 179-183. 